\title{
Optimized siRNA-PEG Conjugates for Extended Blood Circulation and Reduced Urine Excretion in Mice
}

\author{
Frank Iversen ${ }^{1, \#, ~ C h u a n x u ~ Y a n g 1, \#, ~ F r e d e r i k ~ D a g n æ s-H a n s e n ², ~ D a v i d ~ H . ~ S c h a f f e r t ~}{ }^{1}$, Jørgen Kjems ${ }^{1 凶}$ and \\ Shan $\mathrm{Gao}^{1 凶}$
}

1. Interdisciplinary Nanoscience Center (iNANO) and Department of Molecular Biology and Genetics, C.F. Møllers Alle, Building 1130, 8000 Aarhus C, Aarhus University.

2. Department of Biomedicine, Aarhus University, Bartholin Building Building 1240, Wilhelm Meyers Alle 4,8000 Aarhus C, Denmark.

\# Contributed equally.

$\triangle$ Corresponding author: Department of Molecular Biology and Genetics, Aarhus University, C.F. Møllers Alle, Building 1130,8000 Aarhus C, Denmark. Email: jk@mb.au.dk or shg@mb.au.dk; Telephone: 0045 87154975; Fax: 004586196500.

(C) Ivyspring International Publisher. This is an open-access article distributed under the terms of the Creative Commons License (http://creativecommons.org/ licenses/by-nc-nd/3.0/). Reproduction is permitted for personal, noncommercial use, provided that the article is in whole, unmodified, and properly cited.

Received: 2012.12.19; Accepted: 2013.02.06; Published: 2013.02.25

\begin{abstract}
Some of the main concerns with in vivo application of naked small interfering RNA are rapid degradation and urinary excretion resulting in a short plasma half-life. In this study we investigated how conjugation of polyethylene glycol (PEG) with variable chain length affects siRNA pharmacokinetics and biodistribution.

The PEG chains were conjugated to chemically stabilized siRNA at the 5' terminal end of the passenger strand using click chemistry. The siRNA conjugate remained functionally active and showed significantly prolonged circulation in the blood stream after intravenous injection. siRNA conjugated with $20 \mathrm{kDa}$ PEG (PEG20k-siRNA) was most persistent, approximately $50 \%$ PEG20k-siRNA remained th post-injection, while the uncoupled siRNA was rapidly removed $>90 \%$ at $15 \mathrm{~min}$. In vivo fluorescent imaging of the living animal showed increased concentration of siRNA in peripheral tissue and delayed urine excretion when coupled to PEG 20k. Biodistribution studies by northern blotting revealed equal distribution of conjugated siRNA in liver, kidney, spleen and lung without significant degradation $24 \mathrm{~h}$ post-injection. Our study demonstrates that PEG conjugated siRNA can be applied as a delivery system to improve siRNA bioavailability in vivo and may potentially increase the efficiency of siRNA in therapeutic applications.
\end{abstract}

Key words: siRNA, PEGylation, blood circulation, urine excretion, in vivo imaging.

\section{Introduction}

RNA interference (RNAi) is a cellular mechanism, which regulates the expression of genes. RNAi based therapy has demonstrated promising advantages but a main concern for siRNA is delivery in vivo. A particular issue is how to increase siRNA stability in serum, prolong its blood circulation and reduce urine excretion, so a large proportion of drug can reach the target cells in the body. Chemical modification of the siRNA backbone has resulted in increased stability and reduced immune reactions, in- creased specificity and even enhanced silencing effects [1-4]. Aptamers and peptides are commonly conjugated to siRNA for in vivo application to improve target site delivery as well as to assist in translocation across the cell membrane [5-8]. However, if the conjugates are small in relation to the glomerular barrier in the kidneys, they will exhibit reduced circulation time and minimal exposure to the target before they are excreted [9].

Conjugation of poly ethylene glycol (PEG) 
groups to molecules, a technique termed PEGylation, is widely used to improve in vivo pharmacokinetics of protein and chemical drugs, and represents a potential strategy to improve systemic siRNA delivery. PEGylation was first described in the 1970's [10] and there are now numerous PEGylated proteins and aptamers on the pharmaceutical market. Due to the chemical nature and the hydrodynamic size of PEG, PEGylation can lead to increased stability, reduced immunogenicity and prolonged circulation time [11]. The high water coordination of the polymer increases the hydrodynamic volume 3-5 times compared to a globular protein of the same molecular weight and this shell protects against non-specific cell uptake, glomerular filtration and, to some extent, protects the siRNA from enzymatic degradation. Although some studies have been done to test the PEG modified siRNA in vitro [12,13], there is a shortage of detailed work investigating the pharmacokinetics and biodistribution of the PEG-siRNA conjugates in vivo $[12,14]$.

In this study, we aimed to investigate how the length of the PEG chain influences circulation time and urine excretion. A siRNA sense strand, modified with an alkyne at the $5^{\prime}$ end, was conjugated to 5, 10, $20 \mathrm{kDa}$ PEG chains containing a terminal azide group. Additionally, the siRNA overhangs were LNA modified and PEG5k-siLNA, PEG10k-siLNA and PEG20k-siLNA were formed by annealing with the PEGgylated sense strand, respectively.

After annealing and purification, the conjugates were injected into the tail vein of mice. Real-time biodistribution in mice was monitored by an IVIS imaging system, a non-invasive optical imaging technique that provides a dynamic picture of the siRNA biodistribution and urine excretion. Also, RNA was extracted from blood and tissues at different time points and northern blotting was performed to analyze siRNA integrity. We found that in particular the longer PEG chains resulted in extended blood circulation and reduced urinary excretion of the siRNA upon conjugation without eliminating the RNAi function in vitro.

\section{Materials and methods}

\section{I. Materials}

Chemicals and siRNAs: Methoxy-poly (ethylene glycol) derivatives with a terminal azide group (mPEG-Azide, $\mathrm{M}_{\mathrm{w}}$ 5, 10, $20 \mathrm{kD}$ ) were obtained from Nanocs (New York, USA). siRNAs against enhanced green fluorescent protein (EGFP) with locked nucleic acid (siLNA) modification on $3^{\prime}$ - overhang and with $5^{\prime}$ - alkyne or 5'- amino modification was provided by RiboTask APS (Odense, Denmark). The sequence of EGFP siRNA: Hexynyl-5'-GACGUAAACGGCCA
CAAGUtc-3' (sense) and 5'-GGUCAUCCAUGAC AACUUUtt-3' (antisense), small letters denote LNA nucleotides. TBTA (tris[(1-benzyl-1H-1,2,3-triazol-4yl)methyl]amine), sodium ascorbate, $\mathrm{CuSO}_{4}$ were purchased from Sigma-Aldrich (Munich, Germany).

\subsection{Cell lines and siRNA Transfection Rea- gents}

Human lung cancer cell line (H1299) stably expressing EGFP was kindly provided by Dr. Anne Chauchereau (CNRS, Villejuif, France). LipofectamineTM2000, RPMI 1640 culture medium, Penicillin/Streptomycin, Geneticin, Trypsin-EDTA, fetal bovine serum (FBS) and 10×TBE buffer (pH 8.4 \pm 0.10$)$ were purchased from Invitrogen Corporation (Carlsbad, USA). Commercial transfection reagent, TransIT-TKO was obtained from Mirus Corporation (Madison, WI, USA) and Lipofectamine ${ }^{\mathrm{TM}} 2000$ was from Invitrogen (Copenhagen).

\section{3a. Synthesis of siRNA-PEG Conjugates}

siRNA-PEG conjugates were synthesized by employing a 'click chemistry' protocol . In a standard synthesis hexynyl-siLNA and Azido-PEG were dissolved in a 1:1 mixture of $0.1 \mathrm{M}$ phosphate buffer $\mathrm{pH}$ 6.6 and DMSO. TBTA and $\mathrm{CuSO}_{4}$ were dissolved sequentially using the same buffer system, added to the Azido-PEG/siRNA mixture and activated by the addition of an ascorbic acid stock solution. The final concentrations of the various chemicals in the conjugation reaction between mPEG-Azido and alkyne modified siLNA are shown in Table 1 . The reaction was incubated for $3 \frac{1}{2} 2$ hours at room temperature.

Table I. Concentration of the Click Chemistry reagents

\begin{tabular}{ll}
\hline mPEG-Azide & $1 \mathrm{mM}$ \\
\hline Phosphate buffer pH 6.6 & $25 \mathrm{mM}$ \\
Sodium Ascorbate & $20 \mathrm{mM}$ \\
CuSO4 & $400 \mu \mathrm{M}$ \\
Alkyne modified siLNA sense & $125 \mu \mathrm{M}$ \\
TBTA & $3 \mathrm{mM}$ \\
\hline
\end{tabular}

To analyze and purify the conjugates, size-exclusion chromatography (SEC) was performed on an Agilent 1200 Series system using a Phenomenex Biosep-2000 SEC column. Eluents were HPLC-grade TEAA (triethylammonium acetate) buffer $(0.1 \mathrm{M}, \mathrm{pH}$ 7.0). Samples were loaded in TEAA buffer $(0.1 \mathrm{M}, \mathrm{pH}$ 7.0), and fractions collected in 96-well plates. Product containing fractions were pooled and freeze-dried. In order to verify the success of reaction, the various PEG-siRNA conjugates were analyzed using denaturing polyacrylamide electrophoresis $(12 \%$ denaturing polyacrylamide gel, $20 \mathrm{~W}, 60 \mathrm{~min}$ ) 


\section{3b Atto 647N Labeling of siRNAs}

5'-Amino modified siLNAs $(20 \mathrm{nmol}, 10 \mu \mathrm{L})$ were incubated with Atto 647N-NHS $(100 \mathrm{nmol}, 10 \mu \mathrm{L}$ in DMSO) in $60 \mu \mathrm{L}$ of a 1:1 mixture of 0.1 M HEPES ( $\mathrm{pH}$ 7.8) and DMSO for $6 \mathrm{~h}$. The crude dye-labeled siLNAs were precipitated by addition of $10 \mu \mathrm{L} 3 \mathrm{M}$ sodium acetate ( $\mathrm{pH} 5.2)$ and $400 \mu \mathrm{L}$ of a aceton/EtOH mixture (1:1), cooled to $-20^{\circ} \mathrm{C}$ for $1 \mathrm{~h}$ and centrifuged (12000 g, $1 \mathrm{~h}$ ). The resulting pellet was washed three times with EtOH p.a. and dissolved in $40 \mu \mathrm{L}$ nuclease-free water. The oligonucleotides were further $\mathrm{pu}-$ rified by RP-HPLC on an analytical Phenomenex Kinetex XB-C18 column $(150 \times 4.6 \mathrm{~mm})$ using a TEAA/MeCN gradient buffer system (50 mM TEAA $\mathrm{pH}$ : 7.0). The product containing fractions were pooled and freeze-dried.

\subsection{Efficiency of Gene Silencing of PEGylated siRNA in vitro}

Human lung cancer cell line (H1299) stably expressing enhanced green fluorescent protein (EFGP) was used to test the gene silencing efficiency of PEGylated siRNA. Cells were maintained in RPMI media supplemented with $10 \%$ fetal bovine serum, $1 \%$ penicillin-streptomycin, and $1 \%$ Geneticin at $37^{\circ} \mathrm{C}$ in $5 \% \mathrm{CO}_{2}$ and $100 \%$ humidity. For EGFP inhibition studies, cells were plated on 24 -well plates $\left(10^{5}\right.$ cells/well) $24 \mathrm{~h}$ before transfection. siRNA or PEGylated siRNA was mixed with cationic carrier TransIT-TKO or Lipofectamine ${ }^{\mathrm{TM}} 2000$ according to commercial protocol and added at $50 \mathrm{nM}$ final siRNA concentration per well. After overnight incubation, the media was replaced with $500 \mu \mathrm{l}$ fresh cell culturing media. The cells were left for another $24 \mathrm{~h}$ and then harvested using a standard trypsin protocol and resuspended in PBS containing 1\% paraformaldehyde. The EGFP expression level of each sample was measured using Becton Dickenson FACS Calibur flow cytometer. A histogram plot with log green fluorescence intensity (FL-1) on the $\mathrm{x}$-axis and cell number on the $y$-axis was used to define median fluorescence intensity of the main cell population was defined by scatter properties.

\subsection{Fluorescence microscopy}

Transfection efficiency and cellular uptake were analyzed by fluorescence microscopy in human lung cancer cell line (H1299). Transfection was performed as described above, but with Atto $647 \mathrm{~N}$ labeled siRNA for all formulations. Cells were incubated overnight and washed with phosphate-buffered saline (PBS) for 3 times. The fluorescence images were taken using an Olympus IX71 fluorescence microscope and analysed by the CellSense Standard Control Software (ver 1.3 Build 7990).

\subsection{Evaluation of Blood Clearance and Biodis- tribution in mice:}

Balb/c mice ( $25 \mathrm{~g})$ were chosen to investigate the impact of MWs of PEG on siRNA pharmacokinetics. $10 \mu \mathrm{g}$ siLNA duplex in $200 \mu \mathrm{l}$ volumes (corresponding to approximately $\sim 400 \mu \mathrm{g} / \mathrm{kg}$ body weight) either alone or conjugated with PEG5k, PEG10k or PEG20k were administered via tail vein injection $(\mathrm{n}=2$ for each formulation). Blood samples were collected at 1, 15, 30, 60 and 120 min post-injection, and immediately immersed into liquid nitrogen for subsequent RNA isolation. A piece $(\sim 5 \times 5 \times 5 \mathrm{~mm}$ corresponding to 50 to $100 \mathrm{mg}$ ) of kidney, liver, spleen and lung tissue was dissected and transferred into $1.0 \mathrm{ml}$ RNAlater (Qiagen, Denmark) at $24 \mathrm{~h}$ when the experiment was terminated.

Pharmacokinetic studies of PEG20k with $24 \mathrm{~h}$ as ending point were also performed in Balb/c mice ( $25 \mathrm{~g})(\mathrm{n}=4)$. For i.v. administration, $10 \mu \mathrm{g}$ siLNA duplex in $200 \mu \mathrm{l}$ volumes either with or without PEG20k conjugation were injected by tail vein. $30-40 \mu 1$ blood samples obtained by orbital puncture were collected into EDTA tubes, at 1, 5, 15, $30 \mathrm{~min}$ and $24 \mathrm{~h}$ time points. Organs obtained at experimental end point were dissected and stored in RNAlater as described above.

\subsection{Gel Electrophoresis and Determination of siRNA Integrity by Northern Blotting}

Total RNA was purified by Trizol ${ }^{\circledR}$ reagent (Invitrogen) from frozen blood samples and organ tissue in RNAlater. Two micrograms of RNA from blood and $4 \mu \mathrm{g}$ RNA from tissues were run on $15 \%$ denaturing polyacrylamide gels and transferred onto Hybond-N+ membrane (Amersham Biosciences). After UV cross-linking, the membranes were probed with $\left[\gamma^{-32} \mathrm{P}\right]$ ATP labelled sense strand LNA modified siRNA according to standard procedures. The membranes were analyzed on a Typhoon phosphorimager to visualize the antisense strand signal that bound with ${ }^{32} \mathrm{P}$ labeled sense strand of the siRNA [3].

\subsection{In vivo Fluorescence Imaging in Mice using IVIS Imaging System}

Hair from the back and the abdomen of Balb/c mice was removed by shaving to avoid autofluorescence during imaging. Seven hundred fifteen pmol siLNA duplex in $200 \mu \mathrm{l}$ volume either with or without PEG20k conjugation were administered by tail vein injection, PBS was injected as blank control, resulting in a total of 3 groups $(n=2)$. The mice were scanned using an IVIS ${ }^{2} 200$ imaging system (Xenogen, Caliper Life Sciences) under anesthesia with $2.5 \%$ isoflurane. Fluorescence excitation $(\lambda \mathrm{ex}=640 \mathrm{~nm})$ and emission $(\lambda \mathrm{em}=700 \mathrm{~nm})$ filters were used to visualize Atto 
647N labeled siLNAs. Identical illumination settings, including exposure time (1s), binning factor (medium), f-stop (2), and fields of view $(13 \times 13 \mathrm{~cm})$, were used for all image acquisitions. Total emission from inflicted areas (Region of Interest, ROI) of each mouse was quantified with Living Image 4.0 software package. The ratio of average radiant efficiency [(photons $\left.\left./ \mathrm{sec} / \mathrm{cm}^{2} / \mathrm{sr}\right) /\left(\mu \mathrm{W} / \mathrm{cm}^{2}\right)\right]$ in bladder/ in nose was calculated, to compare the urine excretion and blood circulation. The radiance unit of photons $/ \mathrm{sec} / \mathrm{cm}^{2} / \mathrm{sr}$ is the number of photons per second that leave a square centimeter of tissue and radiate into a solid angle of one steradian (sr).

All procedures of animal work were approved by "The Experimental Animal Inspectorate in Denmark" under The Danish Veterinary and Food Administration, Ministry of Food, Agriculture and Fisheries.

\subsection{Statistical Analysis}

Paired Student's T-test was performed for evaluation of knockdown effects in in vitro gene silencing experiment.

\section{Results}

\section{I Synthesis and Characterization of PEGylated siRNA}

Alkyne modified siLNA passenger strand and azide modified PEG chains of different length were coupled using the copper catalyzed Azide-Alkyne Huisgen Cycloaddition (Fig. 1A). After annealing of the guide strand the PEG-siRNA conjugate was purified by size exclusion chromatography (Fig. 1B) and analyzed on a native polyacrylamide gel (Fig. 1C). The migration of the PEG-siRNA conjugates was retarded proportionally with molecular weight of the PEG chain presumably due to the increased hydrodynamic radius and lowered charge density of the construct. All complexes appeared as distinct bands indicating that highly homogenous products are formed (Fig. 1C).

\subsection{Efficiency of Gene Silencing of PEGylated siRNA in vitro}

PEGylation of siRNA may interfere with RNAi potency. In order to investigate the interference of PEGylation on gene silencing, we used both Lipofectamine ${ }^{\mathrm{TM}} 2000$ (Invitrogen, Copenhagen) and TKO-Mirus (Madison, WI) for transfection of PEG-siRNA conjugates in EGFP expressing H1299 cells. The cellular uptake of siLNA alone and all types of PEG-siLNA was comparable (Supplementary Material: Fig. S1). However, the knockdown effects of PEG5k-siLNA, PEG10k-siLNA and PEG20k-siLNA were 60,55 , and $50 \%$, respectively, which were somewhat diminished compared to the knockdown of unmodified siLNA (85-90\%; Fig. 2), but still significantly down regulated compared to the untreated control cells ( $\mathrm{p}<0.05$ or 0.01$)$.

\subsection{Extended Blood Circulation, Decreased Renal Excretion and Wide Organ Distribution of PEGylated siRNA in Mice}

To assess the effect of PEGylation on siRNA half-life in the blood stream, siLNA, PEG5k-siLNA, PEG10k-siLNA and PEG20k-siLNA were injected into mice via the tail vein. We previously demonstrated that unmodified siRNA is rapidly degraded in less than $1 \mathrm{~min}$ after intravenous (i.v.) injection in mice, contrary to siRNA with two 3'-end LNA-modified overhangs (siLNA), which exhibits a half-life of up to 5 min with intact siLNA being detected at least at 30 min post-injection [3] (Fig.3A). In the present study blood samples were collected at 1, 15, 30, 60 and 120 min post injection. In accordance with previous observations (6), siLNA was rapidly removed from the bloodstream exhibiting a $>90 \%$ decline after $15 \mathrm{~min}$ (relative to the $1 \mathrm{~min}$ time point). In contrast, $\sim 50 \%$ of the PEG20k-siLNA remained in circulation after $1 \mathrm{~h}$ and $\sim 15-25 \%$ after $2 \mathrm{~h}$, demonstrating a strong influence of PEG20k on siLNA half-life. PEG10k also had a substantial impact on blood stability of siLNA (PEG10k-siLNA), with $\sim 25 \%$ remaining at $30 \mathrm{~min}$ and $>5 \%$ after $1 \mathrm{~h}$. PEG5k (PEG5k-siLNA) only had a slight effect on siLNA blood levels (Fig. 3A). The siRNA levels and integrity were analyzed in the organs $2 \mathrm{~h}$ post-injection (Fig. 3B). We observed that non-PEGylated siLNA accumulated in kidneys and lungs consistent with previous observations [3] [15]. Conjugation of PEG5k had only little influence on the biodistribution pattern whereas PEG10k-conjugation enhanced the intensity of the siLNA for all four organs. Biodistribution analysis of PEG20k-siLNA yielded a different pattern with spleen being the most strongly targeted organ and a general increase in all other organs. In conclusion, PEG20k-siLNA clearly exhibited the most extended circulation time in the blood stream leading to a more even distribution in different organs. This observation was confirmed by an additional experiment within 4 mice that were terminated at $24 \mathrm{~h}$ post-injection with PEG20k-siLNA. The siLNA was accumulated in four organs with similar intensity (Fig. 3C), with no detectable signal in either the blood samples collected at same time point (Supplementary Material: Fig. S2), or in any organs from mice injected with siLNA alone (mouse 5 and mouse 6 in Fig.3C), indicating that PEG20k could preserve siRNA as an intact molecule within various tissues, even longer than it did in the circulation. 
A

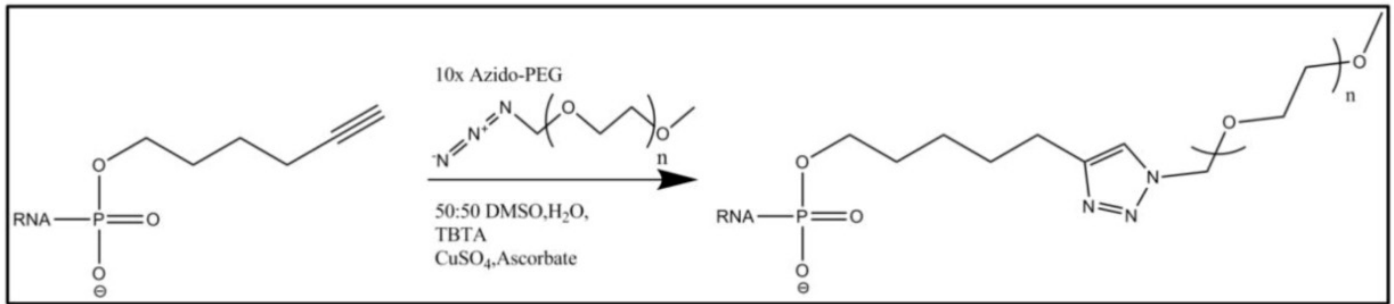

B
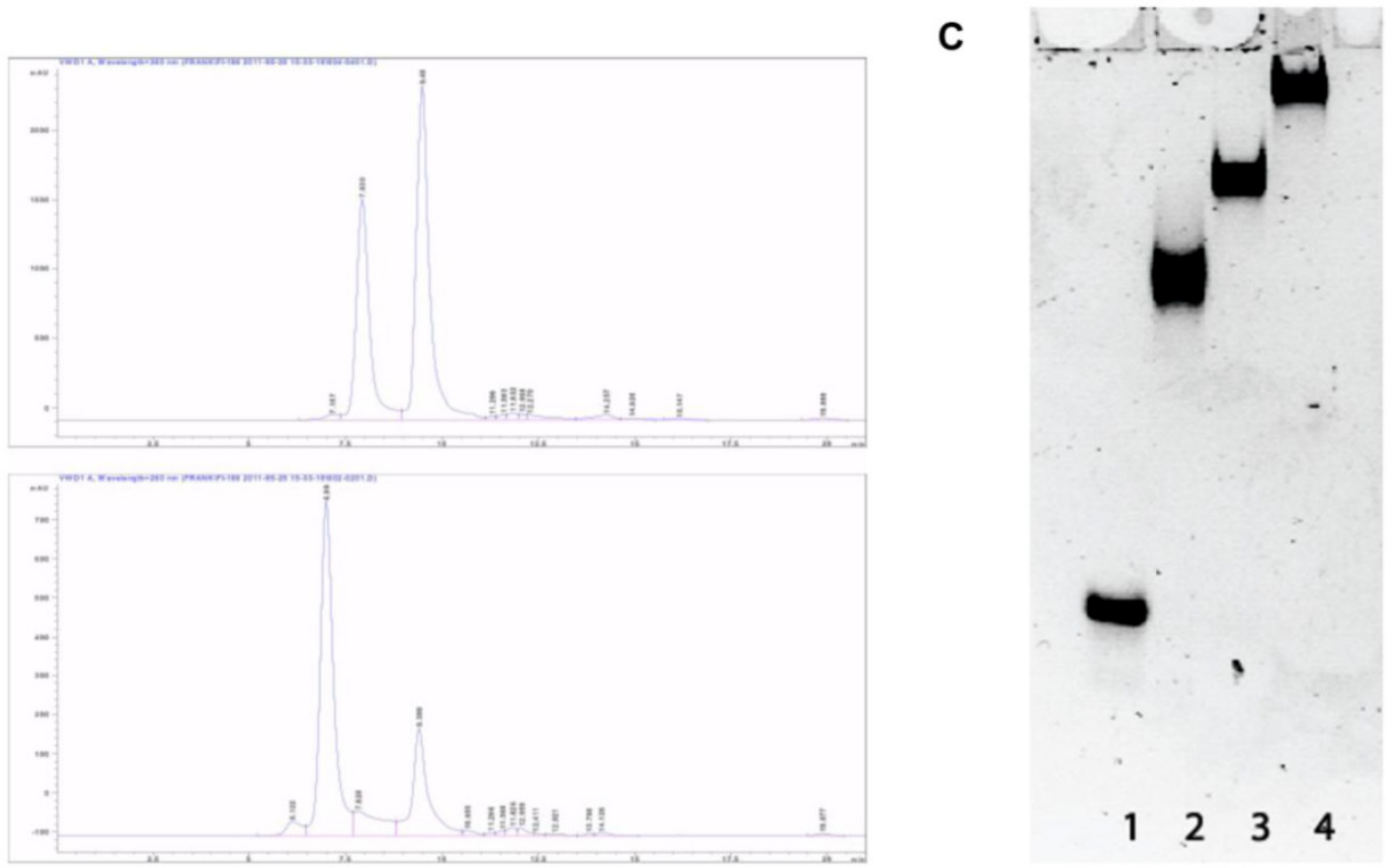

Figure I. Reaction scheme, purification and purity of obtained product. A: Reaction scheme showing the conjugation between siRNA and PEG by Azide-Alkyne Huisgen Cyclo addition. B: Size exclusion HLPC chromatograms after the reaction between siRNA and PEGIOk (left) and PEG20k (right). The first and second peak in both chromatograms represents PEG-siRNA and unreacted siRNA, respectively. C: Analysis of the final conjugation product in $12 \%$ non-denaturing gel polyacrylamide gel. Lane I: naked siRNA, Lane 2 : PEG5k-siRNA, Lane 3: PEGIOk-siRNA, and Lane 4: PEG20k-siRNA.

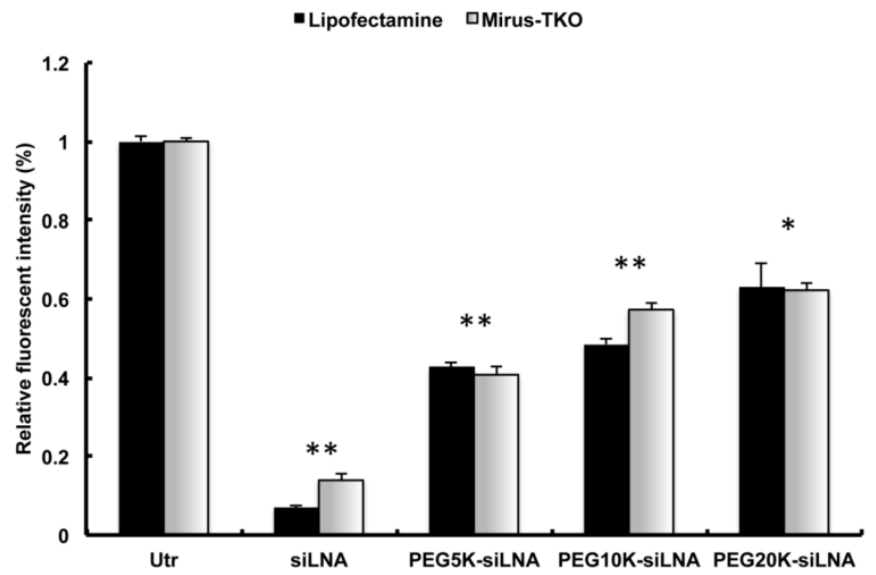

Figure 2. Gene Silencing of PEGylated siRNA in vitro. EGFP knockdown in EGFP expressing HI299 cells by EGFP specific siLNA and siLNA conjugated with different MW PEG and formulated using Lipofectamine ${ }^{\mathrm{TM}} 2000$ or Mirus-TKO. All constructs were applied at $50 \mathrm{nM}$ final concentration of siRNA under serum free condition. EGFP expression was analyzed by flow cytometer and data were obtained from three independent experiments and presented as mean \pm SD $(n=3)$. Significant downregulation of EGFP expression was observed among all treatments $\left(* * p<0.001\right.$ or $\left.*^{*}<0.05\right)$. 
A
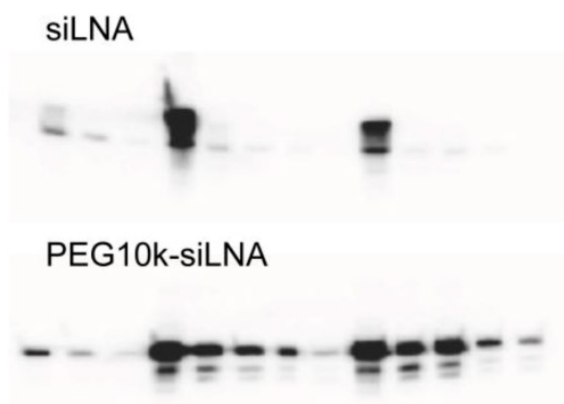

C C1 C2 $11530601201153060120(\mathrm{~m})$

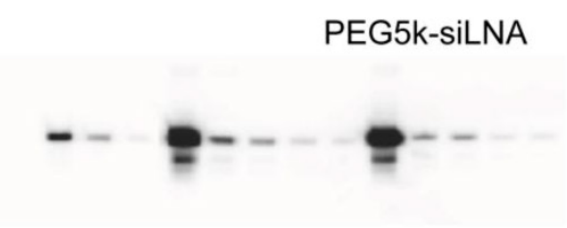

PEG20k-siLNA

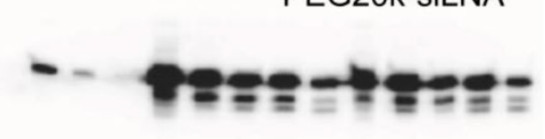

C C1 C2 $11530601201153060120(m)$

B
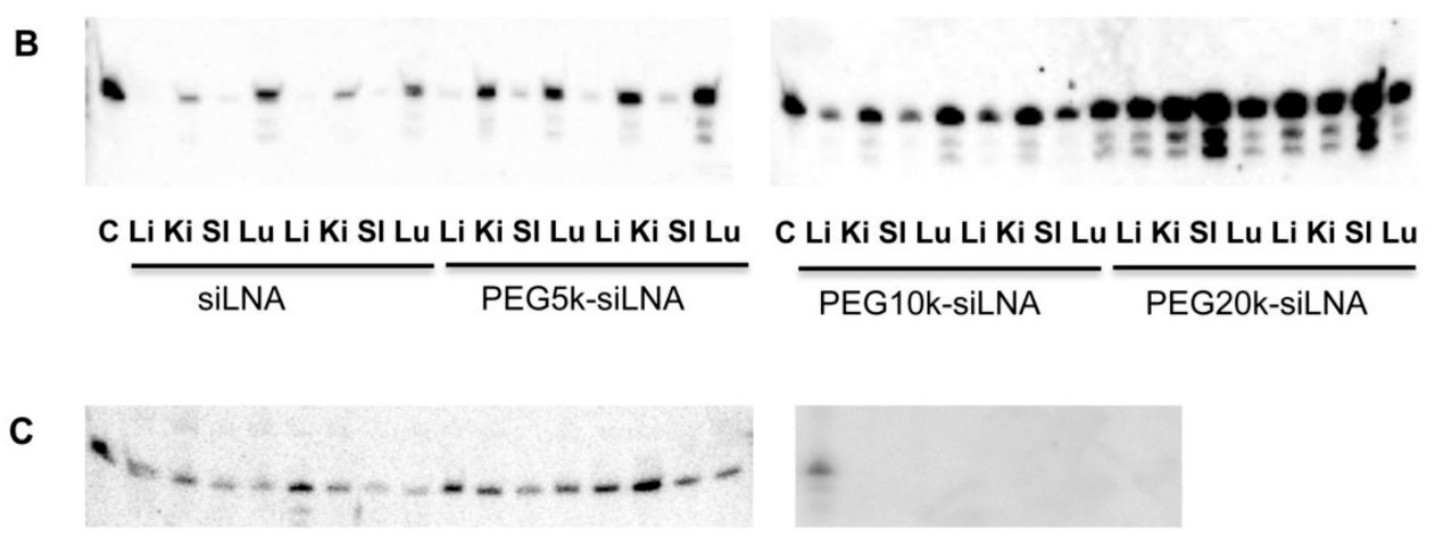
C Li Ki SI Lu Li Ki SI Lu Li Ki SI Lu Li Ki SI Lu $\overline{\text { Mouse1 }} \overline{\text { Mouse2 }} \overline{\text { Mouse3 }} \overline{\text { Mouse4 }}$

\section{Li Ki SI Lu Li Ki SI Lu \\ $\overline{\text { Mouse5 }} \overline{\text { Mouse6 }}$}

Figure 3. Influence of PEG MW on siRNA blood clearance and organ distribution in mice. Northern blot was performed for analysis of siLNA, PEG5k-siLNA, PEGI0k-siLNA and PEG20k-siLNA after i.v. injection of $200 \mu$ in mice ( $n=2)$. (A), siRNA stability in blood circulation: The main band, corresponding to intact siRNA, was harvested at the indicated time points post injection. Loading order: lanes I-3 controls, (C, CI and C2) correspond to I, 0.5 and $0.1 \mathrm{ng}$ unformulated siRNA, respectively, for all 4 blots; lanes 3-7 and 8-I2, RNA samples from blood harvested I, I5, 30, 60 and I 20 min post injection from mouse I and 2 in each group, respectively. (B). siRNA biodistribution: Lane I, 0.I ng siRNA (Control); lanes 2-5 and 6-9 are samples obtained from organs (Lu-lung, Sp-spleen, Ki-kidney and Li-liver) from mouse $\mathrm{I}$ and 2 at $2 \mathrm{~h}$, respectively; two blots represented mice injected with siLNA/PEG5k-siLNA, and PEG I0k-siLNA/PEG20k-siLNA, respectively. (C). PEG20k-siLNA biodistribution 24 h postinjection: Lane I, 0.1 ng siRNA (Control); lanes 2-5, 6-9, I0-13 and I4-I7 are samples from organs (lung, spleen, kidney and liver) from mouse I - 4, respectively, and the same order of loading was performed for mouse 5 and mouse 6 which injected with siLNA alone.

\subsection{Tracking siLNA conjugates by in vivo fluo- rescence imaging}

Optical imaging of living animals provides a non-invasive technique that enables real-time monitoring of fluorescently labeled siRNA biodistribution and excretion. In this study we i.v. injected Atto647N labeled siLNA conjugated either with or without PEG20k and scanned the mice from $5 \mathrm{~min}$ up to $24 \mathrm{~h}$ post-injection.

We observed that PEG20k-conjugation significantly retarded the siLNA accumulation in the bladder, peaking at around $3 \mathrm{~h}$ (Fig. $4 \mathrm{~A}$ ) This contrasts with naked siLNA which peaked in the bladder $5 \mathrm{~min}$ post injection (Fig. 4A). The signal in urine was exclusively seen for naked siLNA already at $1 \mathrm{~min}$ after injection (Example shown as Supplementary Material: Fig. S3), clearly demonstrating a significant effect of PEG20k on urine excretion. To quantitate the effect, we calculated the ratio of the average radiant efficiency in bladder vs. nose\&mouth (which is rich in capillary blood vessels). The ratio in the unmodified siLNA treated group was significantly higher than for the PEG20k-siLNA treated group with the most dramatic difference around $1 \mathrm{~h}$ post injection (Fig. 4B). A significant amount of PEG20k-siLNA is still circulating at $3 \mathrm{~h}$ post-injection, indicated by the strong signals from eyes, nose and paws (Fig. 4A). In contrast, the unmodified siLNA signal was only seen in the kidneys further underlining the improved circulation of PEGylated siRNA. 


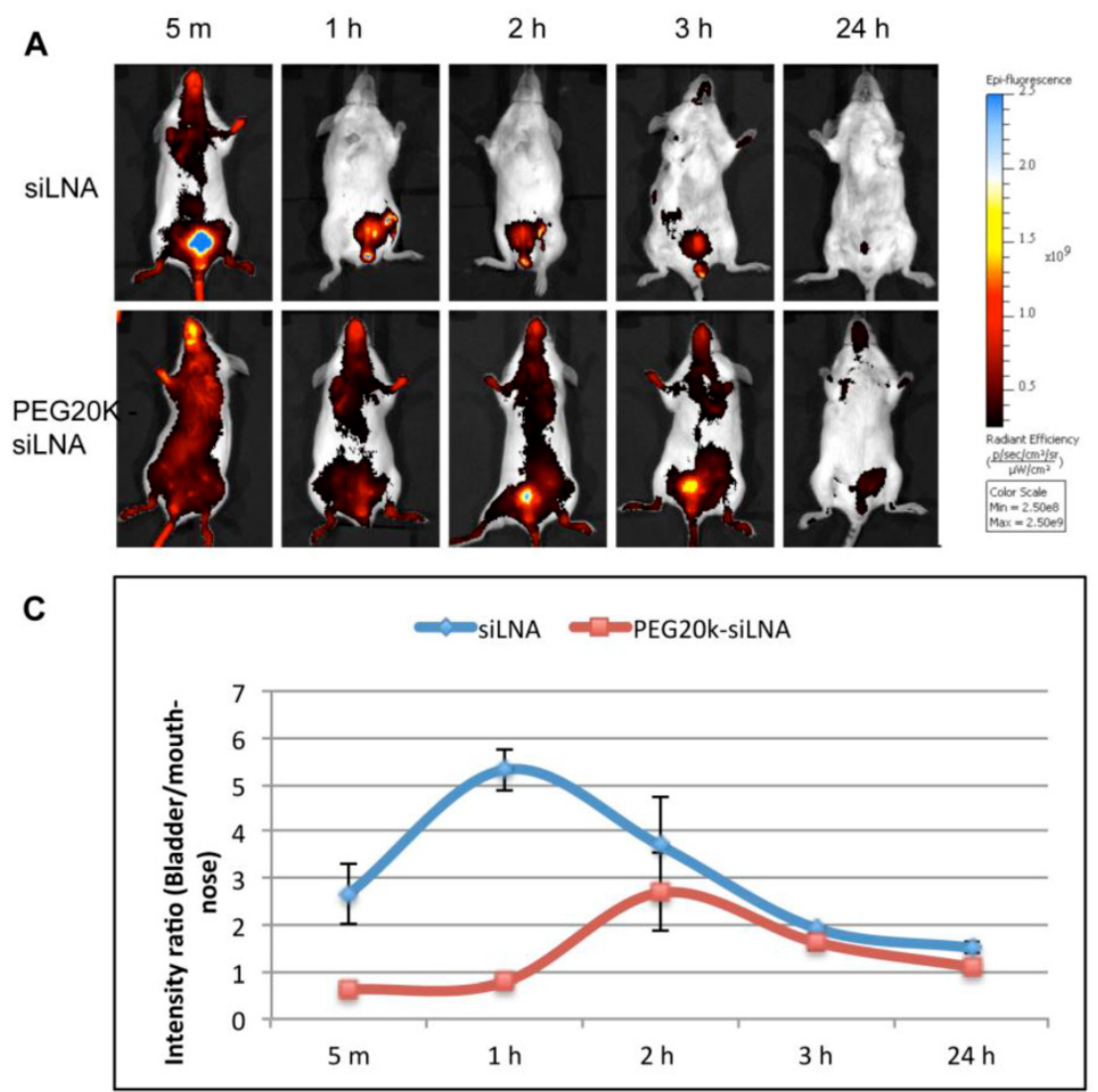

Figure 4. Fluorescence imaging of siLNA and PEG20k-siLNA in live mice. Mice were shaved and fluorophore Atto647N labeled siLNA, Atto647N labeled PEG20k-siLNA and buffer were administered i.v. $(\mathrm{n}=2)$. Fluorescent scanning was performed at the indicated time points post-injection. (A), Columns I-5, images from the ventral side of the mouse at $5 \mathrm{~m}, \mathrm{I} \mathrm{h,} 2 \mathrm{~h}, 3 \mathrm{~h}$ and $24 \mathrm{~h}$, showing the signals at mouth/nose and bladder; (B), images from dorsal side after $3 \mathrm{~h}$, showing signals from paws, ears and kidney. (C), To quantify the signal intensity of images from $4 \mathrm{~A}$, the average radiant efficiency $\left[\left(\right.\right.$ photons $\left.\left./ \mathrm{sec} / \mathrm{cm}^{2} / \mathrm{sr}\right) /\left(\mu \mathrm{W} / \mathrm{cm}^{2}\right)\right]$ was measured using a Living Image 4.0 software package. Ratios of radiance efficiency at the region of interest (ROI) (Bladder/Nose \& Mouth) at the indicated time points are presented.

\section{Discussion}

In the present study we investigated the impact of conjugation of PEG to siRNA in terms of gene silencing in cell culture, blood circulation and biodistribution as well as urinary excretion in mice.

We demonstrated that the use of the copper catalyzed 1,3-dipolar cycloaddition reaction resulted in the successful construction of stable siRNA-PEG conjugates, which was confirmed by both HPLC and gel electrophoresis. Moreover, siRNA bioactivity was conserved during conjugation strategy. We observed a slight inhibition of silencing effect after attaching PEG with longer PEG chains displaying higher inhibition. The silencing potential of siRNA-PEG conjugates has previously been investigated by Jung et al. [13]. Their data also showed a modest $15 \%$ decline in gene silencing efficiency when a PEG5K was attached to the $3^{\prime}$ end of the sense strand via a non-cleavable thioether bond. This impeded silencing is not due to decreased cellular uptake after PEGylation (Supple- mentary Material: Fig. S1), but rather due to the possible interference of PEG chains with the interaction between siRNA-PEG and Dicer as suggested by Jung et al. [13]. Coupling a targeting ligand at the distal end of PEG or introducing a cleavable bond such as disulfide might be able to solve this problem [13].

In our in vivo experiments we found that PEG20k was able to postpone urine excretion, extend circulation time and results in a more even distribution pattern in the various organs.

Prolonged blood circulation is of utmost importance for siRNA delivery in vivo. The life time of unmodified siRNA ranges from $1 \mathrm{~min}$ to $5-6 \mathrm{~min}$ in the bloodstream according to previous reports $[3,16,17]$. To improve the stability of siRNA in blood and to extend its circulation time various options are available: chemical modification on the backbone, conjugation with peptide, antibody or other macromolecules, as well as formulation with non-viral carriers have been widely applied. Conjugation with cholesterol improved half-life with up to $95 \mathrm{~min}$ 
evaluated by radioactivity measurement [16], and $>30$ min by direct gel electrophoresis for pre-labelled siRNA combined with northern blot analysis [3]. The conjugation of PEG can greatly reduce undesired effects such as immune response, unspecific interactions and degradation $[12,13]$, indicating a broad potential in RNAi based therapeutics. In the current study we show that PEG20k can prolong half-life of LNA modified siRNA from $\sim 5$ min up to $1 \mathrm{~h}$ in blood circulation.

Scintillation counting and fluorescence measurements are frequently used to detect the radioactivity or fluorescence signal of labelled siRNA in circulation, due to the challenge of isolating RNA from limited materials e.g. blood samples at multiple time points [17]. However, this is misleading due to contribution of degraded siRNA molecules to the signal. Hence, evaluation of intact siRNA half-life in blood by northern blotting is preferred, because this method can assess both the amount and the integrity of the siRNA $[17,18]$.

When using chemically stabilized siRNA renal excretion is probably the dominating pathway for siRNA disappearance from the blood. In order to improve the circulatory half-life and concomitant siRNA dissemination into the organs [19], it is required to reduce kidney glomerular filtration [20]. Even small chemical modifications can provoke major changes in biodistribution and in vivo bioactivity, e.g. LNA overhang modification, single nucleotide modification by UNA incorporation can significantly increase stability in blood circulation, enhance the nuclease resistance and in vivo gene silencing [4]. Fluorescently labelled siRNA can readily be monitored by in vivo imaging which allows for real time monitoring of whole body siRNA distribution at multiple time points in the same animals, thereby dramatically minimizing the number of mice required. Noninvasive living imaging has been employed previously to assess siRNA delivery in vivo e.g. by positron emission tomography (PET)[21-23], scintigraphic images[24] and fluorescence imaging scanner[25].

In our study living imaging demonstrated that the PEG20k modification significantly delays urine excretion compared to naked siLNA. This result is consistent with previous studies which showed that the fluorescence signal of naked siRNA accumulated in the bladder almost immediately after intravenous administration of siRNA [1,24]. Similarly, siRNA was observed to be rapidly cleared by the kidneys and excreted into urine in mice using Near-Infrared Fluorescence (NIRF) and positron emission tomography (PET) imaging [26].

\section{Conclusion}

The aim of the present study was to investigate the impact of PEG on siRNA pharmacokinetic and biodistribution in mice. Firstly, conjugation of PEG to siRNA was carried out effectively using a click chemistry approach. Secondly, the PEG conjugation was found to prolong circulation time and reduce urine excretion of siRNA. Thirdly, optical imaging could be used to monitor the siRNA circulation and urine excretion in a dynamic fashion and may be useful for a rapid screen of siRNA distribution in vivo. The information gathered is applicable in the design of carrier-free siRNA delivery systems for a successful knockdown in vivo.

\section{Supplementary Material}

Fig.S1 - S3. http://www.thno.org/v03p0201s1.pdf

\section{Acknowledgement}

We thank the Lundbeck Foundation for supporting this work through the grant: Lundbeck Foundation Nanomedicine Center for Individualized Management of Tissue Damage and Regeneration. We thank Rita Rosendahl and Claus Bus for their excellent technical assistance. EGFP expressing cell line H1299 was kindly provided by Dr. Anne Chauchereau (CNRS, Villejuif, France).

\section{Conflicts of Interest}

The authors declare no competing financial interest.

\section{References}

1. Braasch DA, Paroo Z, Constantinescu A, Ren G, Oz OK, Mason RP, Corey DR. Biodistribution of phosphodiester and phosphorothioate siRNA. Bioorg Med Chem Lett 2004;14:1139-1143.

2. Elmen J, Thonberg $\mathrm{H}$, Ljungberg $\mathrm{K}$, Frieden $\mathrm{M}$, Westergaard $\mathrm{M}, \mathrm{Xu}$, et al. Locked nucleic acid (LNA) mediated improvements in siRNA stability and functionality. Nucleic Acids Res 2005;33:439-447.

3. Gao S, Dagnaes-Hansen F, Nielsen EJ, Wengel J, Besenbacher F, Howard KA, Kjems J. The effect of chemical modification and nanoparticle formulation on stability and biodistribution of siRNA in mice. Mol Ther 2009; 17:1225-1233.

4. Laursen MB, Pakula MM, Gao S, Fluiter K, Mook OR, Baas F, et al. Utilization of unlocked nucleic acid (UNA) to enhance siRNA performance in vitro and in vivo. Mol Biosyst 2010;6:862-870.

5. Cesarone G, Edupuganti OP, Chen C, Wickstrom E. Insulin receptor substrate 1 knockdown in human MCF7 ER+ breast cancer cells by nuclease-resistant IRS1 siRNA conjugated to a disulfide-bridged D-peptide analogue of insulin-like growth factor 1 . Bioconjug Chem 2007;18:1831-1840.

6. McNamara JO 2nd, Andrechek ER, Wang Y, Viles KD, Rempel RE, et al. Cell type-specific delivery of siRNAs with aptamer-siRNA chimeras. Nat Biotechnol 2006; 24:1005-1015.

7. Sioud M, Mobergslien A. Efficient siRNA Targeted Delivery into Cancer Cells by Gastrin-Releasing Peptides. Bioconjug Chem. 2012; 23:1040-1049

8. Zhou J, Li H, Li S, Zaia J, Rossi JJ. Novel dual inhibitory function aptamer-siRNA delivery system for HIV-1 therapy. Mol Ther 2008;16:1481-1489.

9. Huang $Y$, Hong J, Zheng S, Ding Y, Guo S, Zhang H, et al. Elimination pathways of systemically delivered siRNA. Mol Ther 2011;19:381-385. 
10. Abuchowski A, Es Tv, Palczuk NC, Davis FF. Alteration of immunological properties of bovine serum albumin by covalent attachment of polyethylene glycol. J Biol Chem 1997;252:3578-3581.

11. Veronese FM, Pasut G. PEGylation, successful approach to drug delivery. Drug Discov Today 2005;10:1451-1458.

12. Kim SH, Jeong JH, Lee SH, Kim SW, Park TG. Local and systemic delivery of VEGF siRNA using polyelectrolyte complex micelles for effective treatment of cancer. J Control Release 2008;129:107-116.

13. Jung S, Lee SH, Mok H, Chung HJ, Park TG. Gene silencing efficiency of siRNA-PEG conjugates: effect of PEGylation site and PEG molecular weight. J Control Release 2010;144:306-313.

14. Lee SH, Mok H, Park TG. Di- and triblock siRNA-PEG copolymers: PEG density effect of polyelectrolyte complexes on cellular uptake and gene silencing efficiency. Macromol Biosci 2011;11:410-418.

15. Moschos SA, Frick M, Taylor B, Turnpenny P, Graves H, Spink KG, et al. Uptake, efficacy, and systemic distribution of naked, inhaled short interfering RNA (siRNA) and locked nucleic acid (LNA) antisense. Mol Ther 2011;19:2163-2168.

16. Soutschek J, Akinc A, Bramlage B, Charisse K, Constien R, Donoghue M, et al. Therapeutic silencing of an endogenous gene by systemic administration of modified siRNAs. Nature 2004; 432:173-178.

17. Malek A, Merkel O, Fink L, Czubayko F, Kissel T, Aigner A. In vivo pharmacokinetics, tissue distribution and underlying mechanisms of various PEI(-PEG)/siRNA complexes. Toxicol Appl Pharmacol 2009;236:97-108.

18. McNamara JO 2nd, Andrechek ER, Wang Y, Rempel KDVaRE, Gilboa E, Sullenger BA, Giangrande PH. Cell type-specific delivery of siRNAs with aptamer-siRNA chimeras. Nat Biotechnol 2006; 24:1005-1015.

19. Lu PY, Xie F, Woodle MC. In vivo application of RNA interference: from functional genomics to therapeutics. Adv Genet 2005;54:117-142.

20. Venturoli D, Rippe B. Ficoll and dextran vs. globular proteins as probes for testing glomerular permselectivity: effects of molecular size, shape, charge, and deformability. Am J Physiol Renal Physiol 2005; 288:F605-13.

21. Medarova Z, Pham W, Farrar C, Petkova V, Moore A. In vivo imaging of siRNA delivery and silencing in tumors. Nat Med 2007;13:372-377.

22. Bartlett DW, Su H, Hildebrandt IJ, Weber WA, Davis ME. Impact of tumor-specific targeting on the biodistribution and efficacy of siRNA nanoparticles measured by multimodality in vivo imaging. Proc Natl Acad Sci U S A 2007;104:15549-15554.

23. Viel T, Boisgard R, Kuhnast B, Jego B, Siquier-Pernet K, Hinnen F, et al. Molecular imaging study on in vivo distribution and pharmacokinetics of modified small interfering RNAs (siRNAs). Oligonucleotides 2008;18:201-212.

24. van de Water FM, Boerman OC, Wouterse AC, Peters JG, Russel FG, Masereeuw R. Intravenously administered short interfering RNA accumulates in the kidney and selectively suppresses gene function in renal proximal tubules. Drug Metab Dispos 2006;34:1393-1397.

25. Li SD, Chen YC, Hackett MJ, Huang L. Tumor-targeted delivery of siRNA by self-assembled nanoparticles. Mol Ther 2008;16:163-169.

26. Hatanaka K, Asai T, Koide H, Kenjo E, Tsuzuku T, Harada N, et al. Development of double-stranded siRNA labeling method using positron emitter and its in vivo trafficking analyzed by positron emission tomography. Bioconjug Chem 2010;21:756-763.

27. Bramsen JB, Laursen MB, Nielsen AF, Bus TBHaC, Langkjaer N, Babu $\mathrm{BR}$, et al. A large-scale chemical modification screen identifies design rules to generate siRNAs with high activity, high stability and low toxicity. Nucleic Acids Res 2009;37:2867-2881. 